\title{
Elías Canales Díaz y la carpintería fina en Valparaíso durante la segunda mitad del siglo XX
}

\author{
FIDEL OLFOS VARGAS
} $>$ Arquitecto. Escuela de Arquitectura, Universidad de Valparaíso, Chile
fidel.olfos@uv.cl

ORCID 0000-0002-7268-104X

Universidad de Valparaíso Facultad de Arquitectura Revista Márgenes Espacio Arte Sociedad Elías Canales Díaz y la carpintería fina en Valparaíso durante la segunda mitad del siglo $\mathrm{XX}$ Diciembre 2021 Vol $14 \mathrm{~N}^{\circ} 21$ Páginas 145 a 151 ISSN electrónico 0719-4436 Recepción mayo 2021 Aceptación agosto 2021 DOI 10.22370/margenes. 2021.14.21.3101

\section{RESUMEN}

Desde hacía años que Marcela veía la dedicación con que su abuelo, Elías Canales Díaz, trabajaba en su taller de carpintería en el Barrio O'Higgins, Valparaíso. Quien fuese la inspiración, "con olor a madera”, que la llevó a estudiar Arquitectura, patrimonio tangible que refleja el espíritu de sus habitantes. He aquí, donde este noble material, proveniente de distintos rincones del planeta, confluyó para levantar edificios tan bellos como los del viejo continente. Don Elías es parte de una generación que vivió los que quizás fueron los últimos años de esplendor de esta ciudadpuerto a mediados del siglo XX, cuando con la llegada a Chile de inmigrantes alemanes y españoles, empresarios habidos de conocimiento y espléndidas maquinarias, se establecieron en El Almendral. A su llegada ven el potencial humano de los pobladores de estos cerros encumbrados, formados en la tradición constructiva del trabajo fino que requieren las herramientas manuales, pero adaptados a una forma de vida dura, exigida por el propio mérito de levantar construcciones en las laderas empinadas. No tardaron en capacitarse, reconociendo el aporte de las máquinas y herramientas eléctricas a su oficio, viendo en este proceso la posibilidad de convertirse en "pequeños industriales".

\section{PALABRAS CLAVE}

patrimonio, madera, tradición, oficio, industrialización

\section{Elías Canales Díaz and fine carpentry in Valparaíso during the second half of the $20^{\text {th }}$ century}

\section{ABSTRACT}

For years, Marcela had seen the dedication with which her grandfather, Elías Canales Díaz, worked in his carpentry workshop in Barrio O'Higgins, Valparaíso. Whoever was the inspiration for her, "with the smell of wood", which led her to study Architecture, a tangible heritage that reflects the spirit of its inhabitants. Here is where this noble material, coming from different corners of the planet, came together to raise buildings as beautiful as those of the old continent. Don Elias is part of a generation that lived through what were perhaps the last years of splendor of this city-port in the middle of the 20th century, when with the arrival in Chile of German and Spanish immigrants, entrepreneurs with knowledge and splendid machinery, they established in El Almendral. Upon arrival they see the human potential of the inhabitants of these lofty hills, trained in the constructive tradition of fine work required by hand tools, but adapted to a harsh way of life, demanded by the merit of building buildings on steep slopes. They were not long in training, recognizing the contribution of machines and power tools to their trade, seeing in this process the possibility of becoming "small industrialists".

\section{KEYWORDS}

heritage, wood, tradition, trade, industrialization 


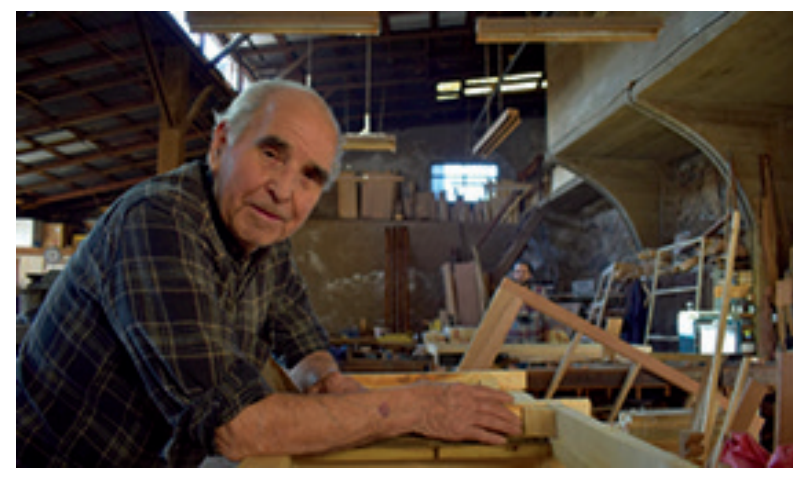

Hay llamas tan profundas que no se extinguen

son capaces de iluminar caminos.

Intenso como un tronco macizo, pellín anclado,

de ramas escultóricamente abiertas, flexibles y elevadas, de raíces desplegadas profundamente,

sensible al tacto, con olor a madera.

Hombre florido,

entre miles de partículas de aserrín,

has sido mi sol y arcoiris.

Apareces absorto,

disfrutando la mágica conexión

del gusto en el hacer.

Concentrado, atento, pausado,

contemplativo, silencioso,

preciso, prolijo, pulcro, dedicado,

perseverante, veloz y enérgico.

Inspirador desde tu actividad y actitud siempre positiva, en la enseñanza del modo de hacer las cosas bien.

A veces buscamos tan lejos,

y lo que necesitamos está en casa,

adentro.

Poema 1

Marcela Canales Oliva, Arquitecta

\section{EL ESTUDIO}

Esta publicación es la primera de una serie de dos publicaciones basadas en una investigación práctica realizada por un grupo de arquitectos de la EAUV, compuesto por Marcela Canales Oliva, Fidel Olfos Vargas, Tomás Bahamondes Alburquenque y Matías Antezana Saavedra, quienes vieron en el trabajo de don Elías Canales Díaz, un cuidadoso cultivo del oficio de la carpintería fina en madera. Con el fin de rescatar su preciado conocimiento práctico deciden postularlo como cultor al Consejo Nacional de la Cultura y las Artes (hoy, Ministerio de la Cultura, las Artes y el Patrimonio), en el marco del llamado Fondart Regional del año 2017, en la línea de Patrimonio Inmaterial: Salvaguardia, reconociendo la relación indisoluble de su oficio con la historia constructiva presente en el patrimonio arquitectónico de la ciudad de Valparaíso.

El sentido del estudio, guiado por el cultor en su taller ubicado en el tradicional Barrio $0^{\prime}$ Higgins, en la avenida Washington $N^{\circ} 1213$, (avenida principal que fuese uno de los primeros caminos a Santiago), consiste en salvaguardar el patrimonio inmaterial presente en las técnicas y destrezas que han sido depuradas por el cultor en la elaboración de objetos de madera en sus 72 años de oficio. Ha sido esencial reconocer y trabajar con madera, conjugando el trabajo artesanal con el industrial, entendiendo la sutileza requerida en el empleo de las herramientas, y el temple, pulso y velocidad en el uso de las máquinas. La buena factura dependerá del tacto y la rigurosidad en la justeza de la ejecución, siendo fundamental la elección del material que entregará sus atributos asociados al uso y la estética.

El registro nace a partir de las palabras, dibujos, fotografías, audios, vídeos y fichas de los procesos de elaboración de estos 5 objetos. Se presenta la biografía del cultor, dado que en su memoria subyace parte de la historia de la ciudad de Valparaíso, desde el ejercicio de este noble oficio.

Se registraron 4 visitas a obras donde trabajó, para apreciar los detalles, reviviendo recuerdos llenos de enseñanzas y reflexiones acerca de la madera como material, los procedimientos en la fabricación y las recomendaciones que son las técnicas especiales o maestrías que dan valor a los objetos confiriéndoles calidad.

Estamos agradecidos por compartir durante este proyecto un tiempo a destiempo, aprendiendo otra vez de la mano de "nuestro abuelo".

\section{BIOGRAFÍA: 4 ETAPAS EN EL OFICIO}

\section{Origen}

Elías Canales Díaz nació el 19 de agosto del año 1928 en la Estrella, un pequeño pueblo de la sexta Región de Chile donde vivió hasta sus 6 años de edad. Tras fallecer su padre, se trasladaría a la ciudad de Valparaíso junto a su madre Sofía Díaz Yáñez y sus dos hermanos mayores Guillermo y Osvaldo. Llegarían a vivir al Cerro Monjas por un corto periodo de tiempo, para luego establecerse en la calle Cantú del Cerro Ramaditas, lugar en donde su madre se dedicaría a ser lavandera.

Su infancia estuvo marcada por la voluntad de ayudar a su madre, quien le haría hincapié en que siempre hay que elegir hacer el bien. A diario él y sus hermanos recolectaban verduras (berros, pencas, cardo santo y chaguales) en los sitios con vertientes del cerro, en su mayoría laderas y fondos de quebradas. Lugares de expedición y reconocimiento del territorio para preparar el almuerzo. Recuerda que siempre nos levantábamos temprano para aprovechar el día. 
Elías se encargaba de hacer distintas tareas para sus vecinos como ir de compras, ensacar pasto para alimentar conejos, ir a entregar meriendas y la ropa que lavaba su madre, de esta manera iba ganando algunos centavos para contribuir en su hogar y siempre le alcanzaba el tiempo hasta para jugar a la pelota.

Fue a la Escuela $\mathrm{N}^{\circ} 44$ de Santa Elena, en donde por su práctica y persistencia lo elegirían para recitar en los actos que se presentaban en el Estadio O'Higgins. Recibiría el premio al mejor compañero y por dicho premio le regalarían un terno del bazar El Cacique, ubicado en el Barrio Puerto de la ciudad.

Tempranamente debió abandonar los estudios para poder seguir apoyando a su familia, por lo que a los 12 años comenzaría a trabajar en el almacén de abarrotes ubicado en el inicio de la avenida Washington del Barrio O ${ }^{-}$Higgins y a sus 17 años en la tienda Ayub Hermanos, donde comenzaría barriendo y luego de un año terminaría siendo vendedor. Además, sería director, tesorero y fisher (organizador de campeonatos) de la asociación de Fútbol Bernardo o`Higgins y árbitro amateur de primera división.

En esta etapa de su vida se daría cuenta que siempre hay algo que se puede hacer, y si esta hecho se puede mejorar, por lo que, si se proponía un objetivo con dedicación y constancia, lo conseguiría. Trabajando en la tienda no podía seguir surgiendo, ante lo cual le pide a su hermano mayor Guillermo, un muy buen mueblista que trabajaba en el cerro Mariposa, que le enseñase el oficio, diciéndole que él quería aprender, pero quería aprender bien.

\section{Formación}

En el año 1945, su hermano mayor lo guiaría y llevaría a trabajar como oficial aprendiz de la fábrica y mueblería fina Beye, ubicada en la calle Colón esquina calle Canciani, la cual tenía su sala de ventas en la calle Condell, frente al edificio consistorial de la llustre Municipalidad de Valparaíso. Este sería el taller donde Elías aprendería a hacer cajones con ensambles cola de Milano, cubiertas, patas $y$, en general, todas las piezas que componen los muebles y su acabado. A diario estudiaba los planos de mobiliario y construcciones llevándoselos a su casa para entenderlos a cabalidad. En esta fábrica fue donde pulió su mano para hacer muebles de estilo, calidad y diseño.

Luego de unos meses se iría a trabajar en la fábrica de muebles de cocina Staub, ubicada en la antigua calle Deformes donde se ubica actualmente el Congreso Nacional. Aquí aprendería a hacer amoblado completo de cocina, muebles bases y superiores.

Al poco tiempo continuaría su aprendizaje y formación en la fábrica de muebles de Ángel Benedicto, ubicada en calle Blanco esquina calle Carrera, donde entraría como oficial adelantado y ascendido rápidamente a Maestro de primera. En esta etapa de su vida aprendería y practicaría su oficio llegando a la pericia y destacaría por ser habilidoso, trabajador, estudioso, perseverante, respetuoso, honrado, responsable y puntual por lo que recibiría todos los años de trabajo dependiente premios e incentivos.

Comenta que le solicitaron fabricar y tallar un amoblado Reina Ana completo (mesa, sillas, aparador, etc.), un encargo importante para el devenir económico de la fábrica por lo que accedería, aunque nunca había tallado se propuso que podía hacerlo. El trabajo fue recibido conforme, lo que le significaría ser considerado y elegido jefe de la fábrica, al presentarse la vacante unos meses más tarde. Fue elegido, siendo el más joven de los maestros que postularon,
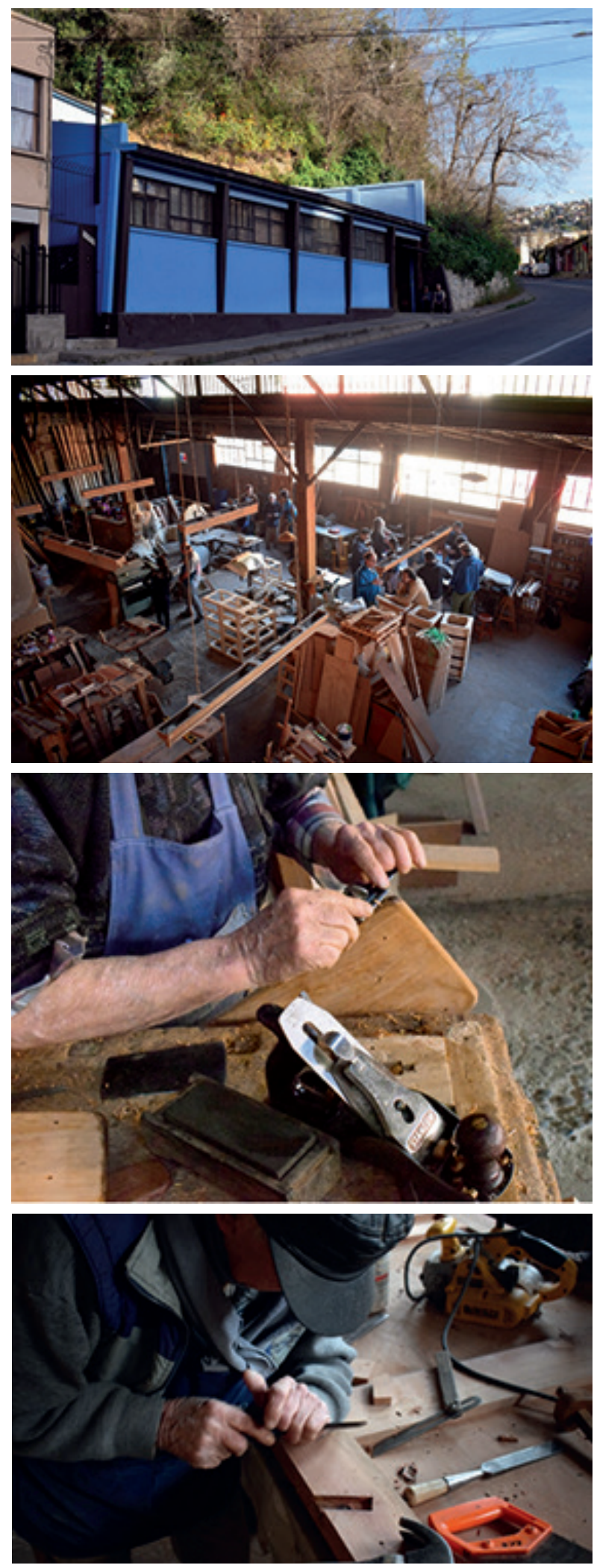

Figura 2. Taller de avenida Washington $N^{\circ} 1213$, Barrio $0^{`}$ Higgins. Fuente: Registro propio.

Figura 3. Taller participativo en el Taller año 2017 de don Elías. Fuente: Registro propio.

Figura 4. Trabajo de pulido con raspador, espauce y cepillo. Fuente: Registro propio.

$>$ Figura 5. Don Elías practicando un inserto en un batiente de puerta. Fuente: Registro propio. 


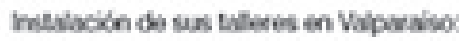

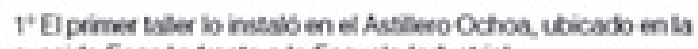
avenida Espora berle a la Esousla houstid.

\section{2" El segundo taler lo insibb en uns casa ques arendsba en ef ceno Elthe.}

$3^{7}$ Su tercer y defintivo taler bo instabo en un terseno que adquibo en enero del aho 1960 en anerida Washingen $N^{2} 1213$. en el berrio Origoins.

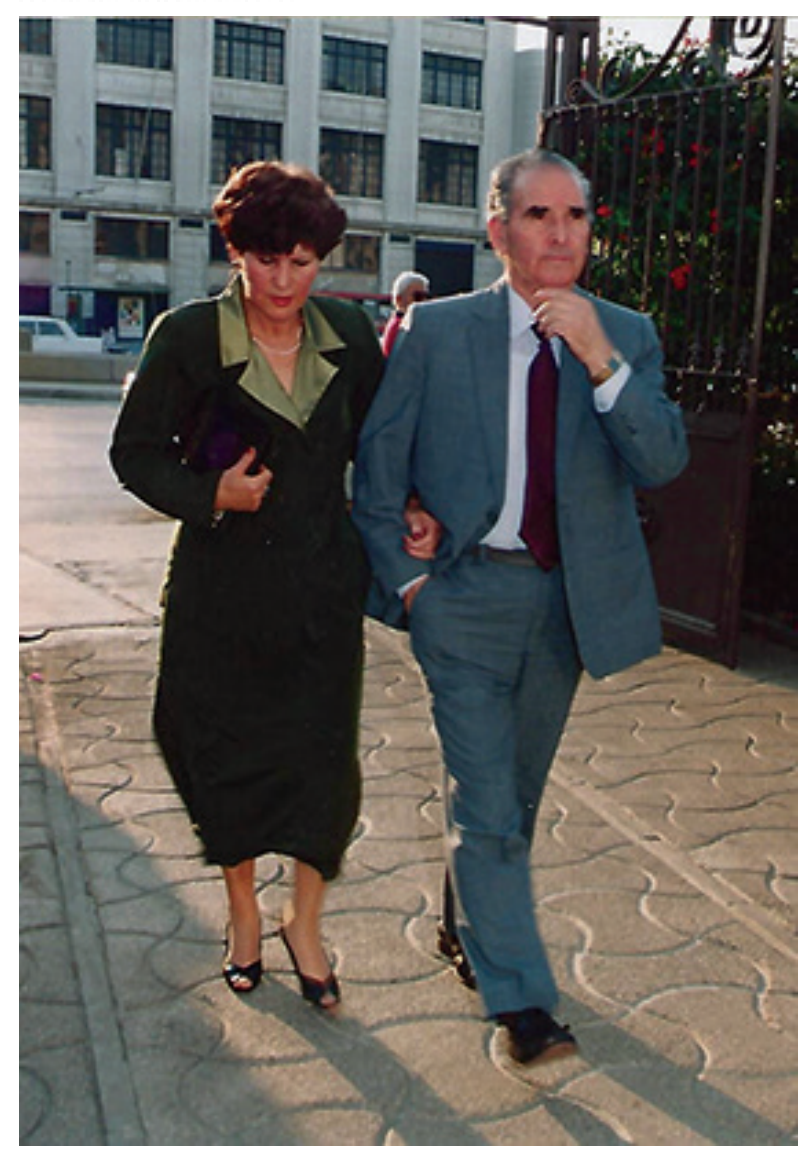

$>$ Figura 6. Plano de Valparaíso con los Talleres que tuvo el cultor. Fuente: Elaboración propia.

> Figura 7. Don Elías y Flor Fernández, su esposa, ingresando al Liceo Juana Ross, en la década de los 80'. Fuente: Archivo familiar del cultor.

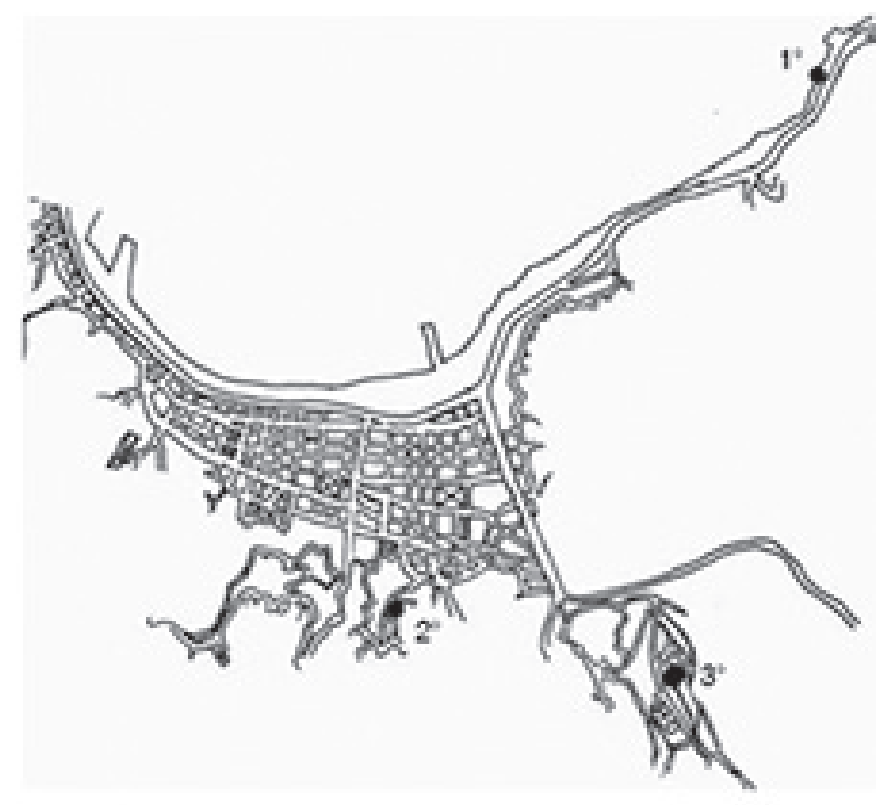

sin llevar currículo, haciéndose cargo de las llaves de la fábrica y un total de 60 trabajadores.

A los 28 años conocería al arquitecto Pablo Mondragón García de las Bayonas, quien le aconsejaría que se independizara, dado que con lo que sabía podría dedicarse a la construcción, desde la carpintería en edificios y mobiliario.

\section{Emprendimiento}

El primer taller independiente lo instaló en los Astilleros Ochoa en la avenida España, frente a la Escuela Industrial de Valparaíso.

En el año 1952 se casó con Flor Fernández Onell, su compañera de vida, con quien arrendarían una vivienda en el cerro El Litre donde nacerían sus dos hijos Reinaldo y Sofía. Allí construyó su segundo taller en un pequeño sitio junto a la casa, en donde Flor lo ayudaría y apoyaría desde sus inicios en distintas labores asociadas a su oficio.

En el año 1956 realizaría su primer trabajo independiente que consistió en fabricar el mobiliario completo para realizar el montaje de la Bienal de Arquitectura de ese año, por encargo del arquitecto Pablo Mondragón García de las Bayonas. Este trabajo le abriría las puertas para conocer y trabajar con connotados arquitectos e ingenieros de la época, como Alfredo Vargas Stoller, Alfredo Vargas Herrera, Hugo Rojas Sepúlveda, Teresio Mezzano y Pedro Herrera Véliz, quien es destacado por Elías como "su favorito" por el fino diseño de sus detalles.

En el año 1960 adquirieron un terreno en la avenida Washington $N^{\circ} 1213$ en el Barrio O ` Higgins, donde poco a poco extrajeron decenas de camionadas de tierra para poder construir el taller de carpintería a nivel de calle y su casa en la parte superior, aterrazando la ladera. En este barrio se radicaron junto a su esposa, participando activamente en el grupo de teatro y realizando charlas matrimoniales en la Parroquia Nuestra Señora de Andacollo del cerro Ramaditas, lugar en donde conocerían a su grupo inseparable de amigos.

Elías recuerda que para terminar la construcción de su casa y taller serían varios amigos los que le tendieron la mano. Entre ellos, don Carlos del Valle, un buen cliente dueño de una fábrica de aluminios, le ayudaría con las planchas de todas las cubiertas. Por otro lado, su amigo eléctrico Oscar Oporto Álvarez le preguntó un día porque trabajaba tanto y no surgía, a lo que Elías respondió que 
hacía todo a mano. Así, un día apareció Oporto en el portón del taller con un motor y un eje de serrucho, con los que fabricamos mi primera máquina, convirtiéndome en un pequeño industrial.

\section{Perseverancia}

Al terminar la semana, Oporto lo sorprendería llegando con una sierra de huincha. Más tarde, cuando su amigo construyó su empresa de electricidad, Elías le habilitaría completamente el interior.

El señor Rogelio Pacheco, Director de Servicio de Impuestos Internos, sería quien le enseñaría a cobrar bien calculando el precio justo en los presupuestos. En aquella época se acostumbraba que amigos y conocidos hicieran trueques, de este modo colaborativo iban surgiendo los emprendedores.

En 1971, el taller se transformó en una pequeña empresa familiar en la que trabajaban 40 maestros, donde su hijo Reinaldo Canales Fernández sería su mano derecha encargándose de la administración y abastecimiento de materiales, tanto para el taller como a las obras.

Después del terremoto del año 1985, trabajó en la reconstrucción de la Parroquia Nuestra Señora de Andacollo. Luego de terminarla, lo llamarían del Obispado de Valparaíso para levantar la cúpula de la Catedral de la ciudad.

De este modo lo iban recomendando y adquiriendo prestigio por su desempeño, convirtiéndose en contratista estrella de primera categoría en destacadas instituciones como: La Armada de Chile, Pontificia Universidad Católica de Valparaíso y el Obispado de Valparaíso.

Al mismo tiempo, trabajaría con grandes empresas como Indus Lever y Compañía Chilena de Tabacos lo que lo mantendría en la vanguardia tecnológica de la época, realizando diversos trabajos que marcarían su trayectoria y le otorgarían prestigio a nivel nacional.

\section{OBRAS}

Elías Canales Díaz realizó obras emblemáticas en Valparaíso, Viña del Mar, Concón y Santiago. Debemos comenzar por destacar su casa-taller, ubicada en la avenida Washington N¹213 en el Barrio O’Higgins, Valparaíso. Desde allí desarrolló la mayor parte de su trabajo. El listado de las obras realizadas es extenso, lleno de historias y enriquecedoras experiencias, de las cuales se presentan a continuación una breve selección.

Uno de sus primeros trabajos como dependiente fue instalar las puertas del edificio de la Cooperativa Vitalicia (1946) del arquitecto Alfredo Vargas Stoller, ubicado en la Plaza Aníbal Pinto, el cual destacó por ser en su momento el más alto del país. Trabajó con el arquitecto Renzo Pecchenino (Lukas) en el amoblado del local de Víctor Bolocco ubicado en calle Esmeralda, donde también confeccionó muebles para radio - tocadiscos. Restauró la casa de la familia Dardel y la histórica casona Pumpin, ambas ubicadas en el Jardín Suizo del Barrio O’Higgins, esta última fue declarada Inmueble de Conservación Histórica (ICH) por su importancia para el barrio y la ciudad.

Posterior al terremoto del año 1985, realizó la reparación de la Parroquia Nuestra Señora de Andacollo en el cerro Ramaditas y la recuperación de la Cúpula de la catedral de Valparaíso ubicada en la Plaza Victoria, encargo del arquitecto Arturo Baeza. El año 1987 construyó las dependencias para recibir al Papa Juan Pablo II, bajo el altar construido por la CAP en Rodelillo, por encargo del arquitecto Hugo Rojas Sepúlveda. Confeccionó las puertas laterales de la Iglesia y Seminario de Lo Vásquez. Construcción de la Capilla de
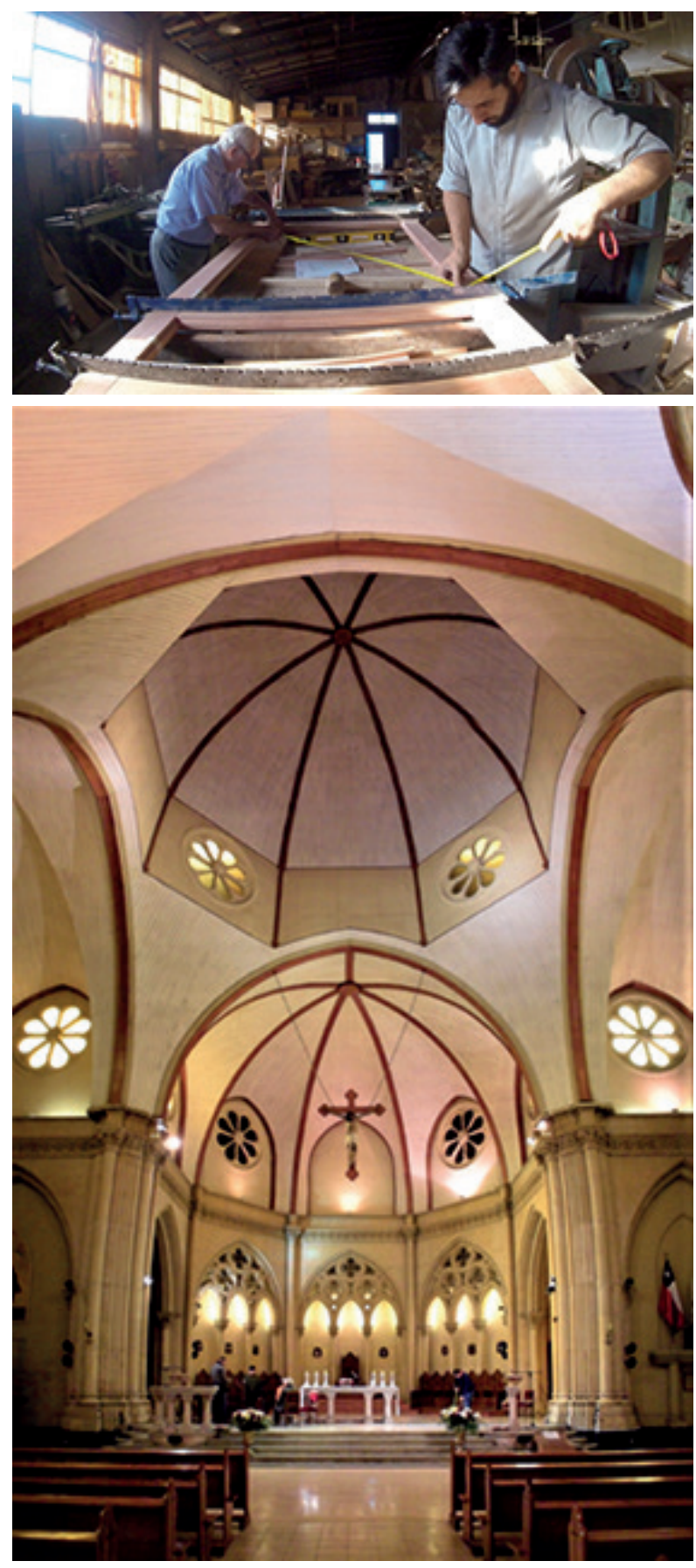

$>$ Figura 8. Don Elías y Tomás Bahamondes, verificando la escuadra de una puerta mampara, en proceso de elaboración. Fuente: Registro propio.

$>$ Figura 9. Vista interior de la cúpula de la Catedral de Valparaíso, el día de la entrevista a don Elías, sentado de espalda, a la izquierda del altar, con motivo del Fondart, año 2018. Fuente: Registro propio. 

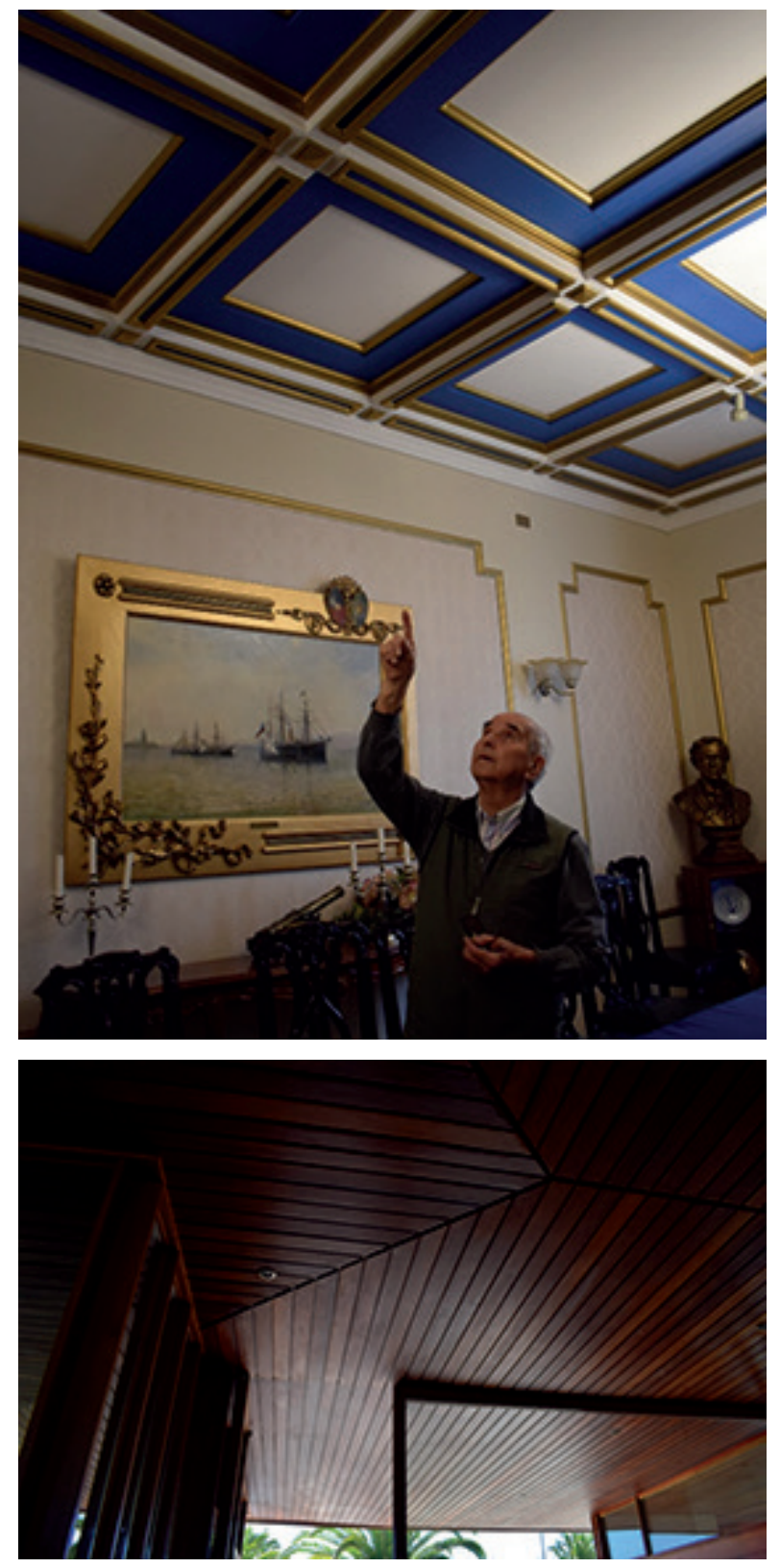

$>$ Figura 10. Comedor Cochrane del Club Naval de Valparaíso, Don Elías señala el trabajo de artesonado diseñado por el arquitecto Pedro Herrera Véliz y elaborado por él. Fuente: Registro propio.

$>$ Figura 11. Fotografía de detalle del cielo, confeccionado con tablas de alerce, para la Cafetería y Administración del Cementerio Parque del Mar, Concón. Fuente: Registro propio.
Quintay. Remodeló casas en OImué por encargo Obispo Emilio Ruiz Tagle Covarrubias.

Confeccionó las divisiones interiores para las oficinas de la Intendencia, trabajo que le valió para realizar además otros encargos, como unas ventanas ojo de buey y puerta de acceso para Isla de Pascua.

Trabajando para la Armada de Chile, recuperó la casa de Arturo Prat en Ningüe, VI Región, por encargo del arquitecto Hugo Rojas Sepúlveda. Remodeló el Club Naval de Valparaíso, por encargo del arquitecto Pedro Herrera Véliz. Confeccionó puertas para la Capilla Naval en Las Salinas. Trabajó en la casa del Almirante Sergio Huidobro Justiniano en 10 Norte, Viña del Mar.

Confeccionó e instaló el montaje de dos Bienales de Arquitectura en el Sporting Club y una en la Sede del Colegio de Arquitectos ubicada en calle Arlegui frente a la Plaza de Viña del Mar.

Para Carabineros de Chile construyó el Polígono de Entrenamiento y el Cuartel de Carabineros de las Fuerzas Especiales ubicado en la ruta 68. Además de la reparación del Retén en Playa Ancha y casas de carabineros en calle Márquez, Valparaíso.

Trabajó en la renovación de la calle Valparaíso, en Viña del Mar, donde amobló la heladería Timbao y la fuente de soda Cevasco, por encargo del arquitecto Alfredo Vargas Herrera, ambas en Galería Couve, en calle Valparaíso esquina Plaza Sucre. Amobló la Farmacia Viñamarina, por encargo del mismo arquitecto y el Salón de Té Virreina, por encargo arquitecto Héctor Muzio. Amoblado de Panadería Su Pan en el edificio Italia en la calle Valparaíso, Viña del Mar, por encargo de Alfredo Vargas Stoller. Amobló las oficinas de Carozzi y edificio de la Municipalidad de Quilpué, por encargo del ingeniero Teresio Mezzano. Hizo el acabado y terminaciones de la administración y cafetería del Cementerio Parque del Mar en Concón, con el arquitecto José Ríos. Oficina de Indus Lever, planta de jabón Le Sancy y planta de Té Lipton en calle Limache, Viña del Mar. Trabajó en la remodelación de oficina de AFP Santa María, en el $8^{\circ}$ piso de la Torre Santa María en Santiago luego del incendio en 1981.

Amobló la casa del doctor Luis Mondragón por encargo del arquitecto Pablo Mondragón. Amobló un conjunto de 3 casas en el pasaje Traslaviña en Viña del Mar, por encargo arquitecto Alfredo Vargas Herrera. Construyó 2 casas en Olmué para el Director y el Subdirector de Impuestos Internos, Rogelio Pacheco y Mario Durán. Trabajó también en la reparación completa de la Casa de Piedra, camino costero de Concón, encargado por arquitecto Arturo Baeza, confeccionando las ventanas y puertas faltantes, además de la reparación de artesonado con vigas traídas de Europa. Construyó 5 viviendas en una recuperación de una antigua edificación en la avenida Washington del Barrio O'Higgins.

Trabajó también para varios establecimientos educacionales, donde destaca su trabajo en la remodelación Casa Central de la PUCV, con obras como las oficinas de Contraloría y del Centro de Alumnos, además de los Laboratorios. Se remodeló completo el Instituto de Matemática de la PUCV, en cerro Barón, por encargo ingeniero Teresio Mezzano. Construcción y amoblado del Colegio Saint Dominic en Reñaca, encargado por el constructor Héctor Derurange.

En el área deportiva, confeccionó puertas y ventanas de Club New Crusaders en el Cerro Alegre, diseño del arquitecto Raúl Besoain. Realizó la construcción de la Sede del Club social las Zorras, frente al Estadio Bernardo O’Higgins de Valparaíso. 


\section{REFLEXIÓN}

La madera es aquella que contiene un universo de elementos que han sido determinantes en el comportamiento humano. La madera es la fibra natural que nos ha dado arquitectura y techo; tabique, mueble y hogar.

Valparaíso ha sido madera, de colinas despobladas de árboles desde siempre, las maderas nativas del sur fundaron la ciudad para que más tarde el roble y oregón norteamericano repoblara de maderas la ciudad. Valparaíso entonces, es lo nativo y lo exótico.

Es la ciudad de Elías Canales, el que detenta el conocimiento universal de la madera, que desde la carpintería modela, guía, estructura y produce objetos, y para el maestro son cosas obvias que están en el mundo de la técnica, de la materia prima y de las herramientas. A Elías le inquieta enseñar otras cosas que concluye en sus más de 90 años de edad, como por ejemplo que un buen oficio aplicado no puede ir separado de la honestidad.

Elías es la Escuela de las Maderas que solo pudo tener tal virtud en la época que le tocó vivir: una carpintería desde las herramientas manuales a otra carpintería, con herramientas eléctricas. Esto es lo técnico o escuela racional, la destreza manual le enseñó las técnicas del uso de herramientas eléctricas volantes o de banco.

Pero la carpintería en lo humano y en lo social tiene otra dimensión para el maestro. Es el comienzo sin recursos: sin capital, sin taller. No es pobreza porque es rico en voluntad y su esfuerzo desborda las colinas de Valparaíso. Aserruchar y cepillar a mano kilómetros de maderas templa la geometría del gesto, para que el ojo juzgue a bien lo que hizo la mano. Entonces las herramientas de motor eléctrico eran una novedad de Europa que podría escasamente llegar a Buenos Aires, una necesidad que para la economía del taller era difícil. Sin embargo, con el tiempo y voluntad no fue imposible. Así crece el hombre, el padre, el esposo, el soñador y realizador, y también la economía a escala humana.

En lo que se refiere a este proyecto que da lugar a este libro, se puede concluir que esta experiencia es la manera de cómo se ennoblece la madera, el mueble y la construcción; de cómo es la existencia de techumbre, puerta, casa, y ciudad; de cómo se da el oficio y la carpintería. Este trabajo de investigación y recopilación de técnicas y he rramientas hecho por arquitectos estudiosos y aplicados en estas materias, es una guía necesaria para carpinteros y arquitectos, una guía para el oficio y el diseño, que nos ilustra sobre la carpintería patrimonial desde la experiencia de vida de un maestro carpintero mueblista. También tiene la autoridad que otorga el conocimiento desde la inteligencia emocional, porque sus autores son la descen dencia de Elías Canales y porque sus autores saben que convivir con maderas es una forma de felicidad.

Marco Meza Figueroa, Escuela Experimental de Artes y Oficios
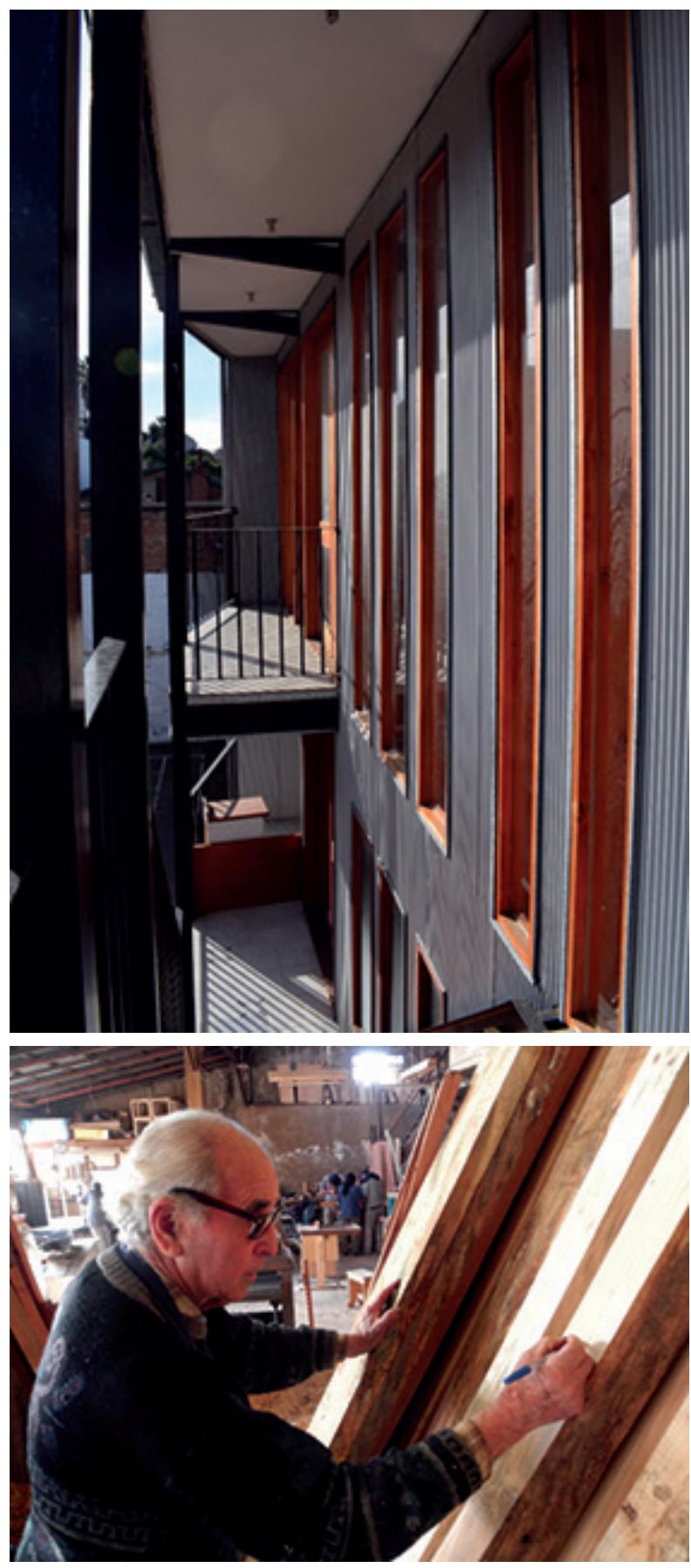

$>$ Figura 12. Fachada con endolados y mampara del Club Deportivo News Crusaders, Cerro Alegre, Valparaíso. Fuente: Registro propio.

$>$ Figura 13. En primer plano, don Elías sacando cuentas sobre la madera, y de fondo, el equipo de arquitectos que desarrolló el estudio, registrando un trabajo ya ejecutado sobre el mesón del cultor. Fuente: Registro propio. 